\title{
The Osgood Criterion and Finite-Time Cosmological Singularities
}

\author{
Ikjyot Singh Kohli \\ isk@mathstat.yorku.ca \\ York University - Department of Mathematics and Statistics
}

May 28, 2022

\begin{abstract}
In this paper, we apply Osgood's criterion from the theory of ordinary differential equations to detect finite-time singularities in a spatially flat FLRW universe in the context of a perfect fluid, a perfect fluid with bulk viscosity, and a Chaplygin and anti-Chaplygin gas. In particular, we applied Osgood's criterion to demonstrate singularity behaviour for Type 0/big crunch singularities as well as Type II/sudden singularities. We show that in each case the choice of initial conditions is important as a certain number of initial conditions leads to finitetime, Type 0 singularities, while other precise choices of initial conditions which depend on the cosmological matter parameters and the cosmological constant can avoid such a finite-time singularity. Osgood's criterion provides a powerful and yet simple way of deducing the existence of these singularities, and also interestingly enough, provides clues of how to eliminate singularities from certain cosmological models.
\end{abstract}

\section{Introduction}

The Osgood criterion [1] is a classical criterion, due to W.F. Osgood in 1898 which gives conditions for ordinary differential equations to admit unique solutions and also to have singularities where solutions explode in a finite time. Following the conventions in [2, Osgood's criterion states a solution $x(t)$ of the initial value problem

$$
\begin{aligned}
\dot{x} & =f[x(t)], \\
x\left(t_{0}\right) & =\xi,
\end{aligned}
$$

blows up in finite time if and only if

$$
\int_{\xi}^{\infty} \frac{d s}{f(s)}<\infty
$$

This condition can be derived from Barrow's formula [3], which says that the solution $\phi(t)$ of such an ODE is given by solving

$$
t-t_{0}=\int_{\xi}^{\phi(t)} \frac{d s}{f(s)} .
$$


Suppose the solution becomes singular in a finite time, $t_{s}$, that is, $\phi\left(t_{s}\right)=\infty$, where $t_{s}<\infty$, then we have that:

$$
t_{s}=\int_{\xi}^{\infty} \frac{d s}{f(s)}+t_{0}<\infty .
$$

Further, a solution to Eqs. (1)-(2) is unique if

$$
\int_{\xi}^{\phi(t)} \frac{d s}{f(s)}=\infty .
$$

Certainly, this is a criterion that is of tremendous importance for physical applications, and as we show in this paper, it is of particular importance in cosmology. In cosmology, assumptions of a spatially homogeneous spacetime, allow Einstein's field equations to be written as an autonomous system of ordinary differential equations. Divergent solutions, that is, solutions that "blow up" in a finite time are indicative of finite-time physical and curvature singularities that occur in universe models. As we show below, Osgood's criterion provides a powerful and yet simple way of deducing the existence of these singularities, and also interestingly enough, provides clues of how to eliminate singularities from certain cosmological models. Despite this, we note that Osgood's criterion has received very little attention in the physics and cosmology community. For this reason, we believe that the work here is unique, and could be of interest in future cosmological studies.

In this paper, we will attempt to study the question of whether an expanding spatially flat Friedmann-Lemaîrte-Robertson-Walker (FLRW) universe with cosmological constant and ordinary and exotic matter will expand forever in the future or develop a finite-time singularity. It is understood that the ultimate fate of the universe depends on possible decay of dark energy in the future, and if it does not occur, the universe will expand forever [4].

Further, the concept of the existence of singularities in the context of general relativity has been studied quite extensively. With respect to the cosmological case, there is the fundamental singularity theorem [4] 5] 6] which describes irrotational geodesic singularities, and states that if $\Lambda \leq 0, \mu+3 p \geq 0$, and $\mu+p>0$ in a fluid flow where $\mu$ is the energy density of the fluid, $p$ is the pressure of the fluid, in addition to having $\dot{u}=0, \omega=0$, and $H_{0}=0$ at some time $s_{0}$, then a spacetime singularity, where either the expansion scalar goes to zero or the shear scalar diverges occurs at a finite proper time $\tau_{0} \leq 1 / H_{0}$ before $s_{0}$.

Further elaborations are built upon this singularity theorem. It is of interest to note that in 4 ] five possible routes to avoid the conclusions of this singularity theorem are discussed in detail. They are a positive cosmological constant, acceleration, vorticity, an energy condition violation, or alternative gravitational equations. We refer the interested reader to 4 for further discussions regarding these issues.

Following [4, we also note that singularities occur in cosmology not only in the context of FLRW models, but also for realistic anisotropic and inhomogeneous models of the universe in which the strong energy condition $\mu+3 p>0$ is satisfied. Related to this, Penrose [7] did pioneering work on black hole singularities producing a theorem that allowed one to predict the existence of singularities in realistic gravitational collapse cases. Hawking [8] extended these results to the cosmological context leading to the famous Hawking-Penrose singularity theorem which implied that space-time singularities are to be expected if either the universe is spatially closed or there is an 'object' undergoing relativistic gravitational collapse (existence of a trapped surface) or there is a point $p$ whose past null cone encounters sufficient matter that the divergence of the null rays through $p$ changes sign somewhere to the past of $p$ (i.e. there is a minimum apparent solid angle, 
as viewed from $p$ for small objects of given size). The theorem applies if the following four physical assumptions are made: (i) Einstein's equations hold (with zero or negative cosmological constant), (ii) the energy density is nowhere less than minus each principal pressure nor less than minus the sum of the three principal pressures (the 'energy condition'), (iii) there are no closed timelike curves, (iv) every timelike or null geodesic enters a region where the curvature is not specially aligned with the geodesic. (This last condition would hold in any sufficiently general physically realistic model.)

Further, Bekenstein [9] studied exact solutions of Einstein-conformal scalar equations and presented a class of FLRW models which contained both incoherent radiation and a homogeneous conformal scalar field which bounce and never pass through a singular state, thereby circumventing the singularity theorems by violating the energy condition. Parker and Fulling [10] considered a classical gravitational field minimally coupled to a quantized neutral scalar field possessing mass. They concluded that quantum effects can sometimes lead to the avoidance of the cosmological singularity, at least on the time scale of one Friedmann expansion. Collins and Ellis [11] examined in detail the singularities that occur in Bianchi cosmologies. Barrow and Matzner [12] showed that the singularity corresponding to homogeneous and isotropic universes observationally equivalent to ours must be of simultaneous Robertson-Walker type containing only small curvature fluctuations. In an interesting application of Robertson-Walker singularities, Barrow [13 showed that the large-scale velocity and vorticity fields required for the vortex and spinning-core theories of galaxy formation can be generated primordially in a natural way from a FLRW singularity.

Related to future finite-time singularities that develop in FLRW models is the question of the so-called "ultimate fate of the universe", there are several possibilities [14] which we list in Table1.

\begin{tabular}{|c||c||c||c|}
\hline Singularity Type & Description & $T$ & $K$ \\
\hline Big Crunch (Type 0) & $\theta, p, \mu \rightarrow \infty, l \rightarrow 0$ & Strong & Strong \\
\hline Big Rip (Type I) [15] [16] & $l, p, \mu \rightarrow \infty$ & Strong & Strong \\
\hline Sudden Singularities (Type II) [17]37] & $l, \theta, \mu<\infty, p \rightarrow \infty$ & Weak & Weak \\
\hline Big Freeze (Type III) [38] & $l<\infty, \theta, \mu, p \rightarrow \infty$ & Weak & Strong \\
\hline Generalized Sudden / Big Separation (Type IV) [39] & $l, \theta, \mu, p<\infty, \dot{p} \rightarrow \infty$ & Weak & Weak \\
\hline$w$-Singularities (Type V) [40[42] & $l<\infty, \mu, p \rightarrow 0, w \rightarrow \infty$ & Weak & Weak \\
\hline
\end{tabular}

Table 1: A classification of known finite-time singularities. $l$ refers to the scale factor in the FLRW metric, $p$ and $\mu$ refer to the pressure and energy density of the matter content in the FLRW spacetime, $w$ is the barotropic index, and $\theta$ is the expansion scalar as defined above. Note that, sudden singularities are weak singularities where the spacetime can be extended after the singular event. The singularities are classified as being strong or weak according to the classification due to Tipler (T) 43] and Krolak (K) 44].

Further, so-called infinite-time singularities may also occur as well. These include Type $\infty$ or directional singularities, which are of strong type according to both 43 and 44 . There are also other types of non-singular future behaviour which are also of the infinite-time variety. These include the little rip [45, 46, pseudo-rip [47, and the little sibling of the big rip [48. These are discussed in more detail in [49] and [14].

It is also important to note that conditions describing the avoidance of finite-time singularities have been given in [18, 50, 53.

In this work, we will consider a spatially homogenous and isotropic $k=0$ FLRW universe with a cosmological constant and different types of matter sources. We will use Osgood's criterion to 
establish when such models admit finite-time, Type 0 singularities as discussed below.

\section{The Dynamical Equations}

It is well-known that covariant derivative of the four-velocity vector $u_{a}$ can be decomposed as 54 [55]

$$
u_{a ; b}=\frac{1}{3} \theta h_{a b}+\sigma_{a b}+\omega_{a b}-\dot{u}_{a} u_{b}
$$

where $u_{a}$ is the fluid four-velocity, $\theta$ is the expansion scalar, $\sigma_{a b}$ is the shear tensor, $\omega_{a b}$ is the vorticity tensor, and $h_{a b}$ is the standard projection tensor. For spatially homogeneous universes, we can choose the four-velocity vector to be orthogonal to the space-like surfaces in the $3+1$-spacetime decomposition. With this, the shear tensor $\sigma_{a b}$, the vorticity tensor $\omega_{a b}$, and acceleration $\dot{u}_{a}$ all vanish. One then takes projected, symmetric, and trace-free components of the decomposition equation above in combination with the Einstein field equations

$$
R_{a b}-\frac{1}{2} R g_{a b}+\Lambda g_{a b}=\kappa T_{a b},
$$

to obtain the Raychaudhuri equation

$$
\dot{\theta}+\frac{1}{3} \theta^{2}+\frac{\kappa}{2}(\mu+3 p)-\Lambda=0,
$$

the energy density evolution equation

$$
\dot{\mu}+\theta(\mu+p)=0,
$$

and the generalized Friedmann constraint equation

$$
\frac{1}{3} \theta^{2}=\kappa \mu+\Lambda,
$$

where $\mu$ denotes the matter energy density and $p$ denotes the matter pressure.

In fact, we have that by the Friedmann equation (11),

$$
\lim _{t \rightarrow t_{s}} \theta_{t}=\infty \Rightarrow \lim _{t \rightarrow t_{s}} \frac{1}{3} \theta^{2}=\infty \Rightarrow \lim _{t \rightarrow t_{s}}[\kappa \mu(t)+\Lambda]=\infty \Rightarrow \lim _{t \rightarrow t_{s}} \mu(t)=\infty .
$$

Therefore, the Osgood criterion provides a way of testing for a singularity where $\theta$ and $\mu$ go to infinity in a finite time. This type of singularity is a big crunch or Type 0 singularity. In the context of singularity classification, the Type 0 singularity is considered a strong singularity [43, 44, 49,

The advantage of considering spatially flat models is that the dynamical equations (9), (10) can be reduced to a single dynamical equation via Eq. (11). We will therefore consider the dynamics of such models to be represented by an evolution equation for $\theta$, although, the analysis in what

follows is equally as valid for an evolution equation for $\mu$. Substituting Eq. (11) into Eq. (9), we obtain

$$
\dot{\theta}=-\frac{1}{2}(1+w)\left(\theta^{2}-3 \Lambda\right),
$$

where $w$ is the equation of state parameter. 


\section{The Existence of Finite-Time Singularities}

With the main evolution equation (13) in hand, we now demonstrate the existence of finite-time singularities. To accomplish this, we will make use of Osgood's criterion as defined earlier.

To apply Osgood's criterion to our problem, let us consider the initial value problem

$$
\begin{aligned}
\dot{\theta} & =-\frac{1}{2}(1+w)\left(\theta^{2}-3 \Lambda\right), \\
\theta\left(t_{0}\right) & \equiv \theta_{0} .
\end{aligned}
$$

Following the introductory section and evaluating the integral in (3), we obtain

$$
\begin{aligned}
t_{s}-t_{0} & =\int_{\theta_{0}}^{\infty}-\frac{2 d s}{(1+w)\left(s^{2}-3 \Lambda\right)} \\
& =\frac{1}{\sqrt{3}(1+w) \sqrt{\Lambda}} \log \left|\frac{\theta_{0}-\sqrt{3} \sqrt{\Lambda}}{\theta_{0}+\sqrt{3} \sqrt{\Lambda}}\right| .
\end{aligned}
$$

Note that $t_{s} \rightarrow \infty$ only if $\theta_{0}= \pm \sqrt{3} \sqrt{\Lambda}$, where the positive value is precisely the value of the de Sitter expansion. Therefore, carefully choosing the initial condition to be equal to the rate of the de Sitter expansion avoids a finite-time singularity where $\theta \rightarrow \infty$.

The value of Eq. (16) is finite and real if either

$$
\{\Lambda>0\} \cap\left\{\theta_{0}>\sqrt{3} \sqrt{\Lambda}\right\} .
$$

Hence, for choices of $\theta_{0}$ that satisfy these inequalities, the integral in Eq. (16) is finite, and hence $\theta \rightarrow \infty$ in a finite time.

For the sake of completeness, let us consider the case where $\Lambda<0$. In this case, the integral Eq. (16) is evaluated to be

$$
t_{s}-t_{0}=\frac{\pi\left(-\frac{1}{\Lambda}\right)^{3 / 2} \Lambda^{3 / 2}-2 \tanh ^{-1}\left(\frac{\theta_{0}}{\sqrt{3} \sqrt{\Lambda}}\right)}{\sqrt{3} \sqrt{\Lambda}(w+1)} .
$$

For $\Lambda<0$, this integral is finite and real if and only if $\theta_{0}=0$. Therefore, for this choice of initial condition, this universe model will exhibit finite-time, type 0 singularities. Further, the integral diverges if $\theta_{0}= \pm \sqrt{3} \sqrt{\Lambda}$, which, as mentioned above the positive root is that of the de Sitter expansion. Therefore, a finite-time, type 0 singularity can be avoided by choosing the initial condition to be that of the de Sitter expansion.

\section{FLRW Models With Bulk Viscosity}

We now briefly consider $k=0$ FLRW models with bulk viscosity. Bulk viscous models are typically used in early-universe cosmological models. The role of the bulk viscosity is to simply add a pressure term in the perfect-fluid energy-momentum tensor. The perfect-fluid energy momentum tensor which is imposed by the symmetry of FLRW models for fluid density $\mu$, pressure $p$, and four-velocity vector $u_{a}$ is given by

$$
T_{a b}=(\mu+p) u_{a} u_{b}+g_{a b} p
$$


By adding a bulk viscosity term, this energy-momentum tensor is now $[56$

$$
T_{a b}=(\mu+p) u_{a} u_{b}+g_{a b} p-\xi \theta h_{a b},
$$

where $h_{a b}$ is the projection tensor, and $\xi$ is the bulk viscosity, and $p=w \mu$.

Following [57, we assume an equation of state

$$
\xi=\xi_{0} \mu^{m},
$$

where $m$ is a constant and $\xi_{0}$ is a positive constant.

With this definition in mind, let us define the total effective pressure as

$$
p=\tilde{p}-\xi \theta,
$$

where $\tilde{p}$ is defined as $\tilde{p}=w \mu$. Then, by simply substituting this definition of $p$ into Eq. (11), we see that Eq. (9) becomes

$$
\dot{\theta}=\frac{1}{2}\left[\theta \kappa 3^{1-m} \xi_{0}\left(\frac{\theta^{2}-3 \Lambda}{\kappa}\right)^{m}-\theta^{2}(w+1)+3 \Lambda(w+1)\right] .
$$

It is not possible to apply Osgood's criterion in a general sense to Eq. (23), that is, for an arbitrary $m$ in Eq. (23). We will therefore consider only specific values for $m$ in our analysis. In fact, the choice of $m$ is very important and can affect the overall dynamics of general FLRW models. This point was extensively studied in $57-62$. We will only consider the cases $m=0$ and $m=1$ in what follows as these are physically relevant according to [57] and 62].

\subsection{Case: $m=0$}

This case corresponds to the case of a constant bulk viscosity. The Raychaudhuri equation (23) becomes

$$
\dot{\theta}=-\frac{\theta^{2}}{2}+\frac{3 \theta \kappa \xi_{0}}{2}+\frac{3 \Lambda}{2}-\frac{\theta^{2} w}{2}+\frac{3 \Lambda w}{2},
$$

where we will use $\theta\left(t_{0}\right)=\theta_{0}$ as an initial condition.

Then, Osgood's criterion becomes

$$
t_{s}-t_{0}=\int_{\theta_{0}}^{\infty}-\frac{2 d s}{(1+w) s^{2}-3(1+w) \Lambda-3 s \kappa \xi} .
$$

Evaluating this integral, we find that it is equal to

$$
t_{s}-t_{0}=\frac{2 \log \left|\frac{\sqrt{3} \sqrt{3 k^{2} \xi_{0}^{2}+4 \Lambda(w+1)^{2}}}{3 k \xi_{0}-2 \theta_{0}(w+1)}+1\right|-\log \left|\frac{\sqrt{3} \sqrt{3 k^{2} \xi_{0}^{2}+4 \Lambda(w+1)^{2}}}{2 \theta_{0}(w+1)-3 k \xi_{0}}+1\right|}{\sqrt{3} \sqrt{3 k^{2} \xi_{0}^{2}+4 \Lambda(w+1)^{2}}} .
$$

This integral only diverges for

$$
\theta_{0}=\frac{3 \kappa \xi_{0} \pm \sqrt{3} \sqrt{4(1+w)^{2} \Lambda+3 \kappa^{2} \xi_{0}^{2}}}{2(1+w)}
$$


Therefore, only for the initial condition as given in Eq. (27), does a unique solution exist to Eq. (24) where there is no finite-time singularity where $\theta \rightarrow \infty$. Finite-time singularities therefore exist, for initial conditions $\theta_{0}$ (assuming $-1<w \leq 1, \xi_{0}>0, \Lambda>0$ ) if

$$
\theta_{0}>\frac{1}{2} \sqrt{\frac{9 k^{2} \xi_{0}^{2}+12 \Lambda+12 \Lambda w^{2}+24 \Lambda w}{(w+1)^{2}}}+\frac{3 k \xi_{0}}{2(w+1)} .
$$

\subsection{Case: $m=1$}

The Raychaudhuri equation (23) becomes

$$
\dot{\theta}=\frac{1}{2}\left(\theta^{2}-3 \Lambda\right)\left(-1-w+\theta \xi_{0}\right), \quad-1<w \leq 1, \quad \Lambda>0 .
$$

Specifically, we now wish to apply Osgood's criterion to the initial value problem

$$
\begin{aligned}
\dot{\theta} & =\frac{1}{2}\left(\theta^{2}-3 \Lambda\right)\left(-1-w+\theta \xi_{0}\right), \\
\theta\left(t_{0}\right) & =\theta_{0} .
\end{aligned}
$$

Osgood's criterion then requires us to check the convergence/divergence behaviour of

$$
t_{s}-t_{0}=\int_{\theta_{0}}^{\infty} \frac{2 d s}{\left(s^{2}-3 \Lambda\right)\left(s \xi_{0}-w-1\right)}
$$

Evaluating this integral, we find that it is equal to

$t_{s}-t_{0}=-\frac{2 \sqrt{3}(w+1) \operatorname{coth}^{-1}\left(\frac{\theta_{0}}{\sqrt{3} \sqrt{\Lambda}}\right)-3 \sqrt{\Lambda} \xi_{0}\left(2 \log \left|-\xi_{0}\right|+\log \left|\theta_{0}^{2}-3 \Lambda\right|-2 \log \left|-\xi_{0} \theta_{0}+w+1\right|\right)}{3 \sqrt{\Lambda}\left(-3 \Lambda \xi_{0}^{2}+w^{2}+2 w+1\right)}$.

The integral in (32) has finite real values if

$$
\left\{0<\Lambda<\frac{1+2 w+w^{2}}{3 \xi_{0}^{2}}\right\} \cap\left\{\theta_{0}>\sqrt{3} \sqrt{\Lambda}\right\},
$$

or

$$
\left\{\Lambda \geq \frac{1+2 w+w^{2}}{3 \xi_{0}^{2}}\right\} \cap\left\{\theta_{0}>\sqrt{3} \sqrt{\Lambda}\right\}
$$

Now, the integral in (32) diverges if

$$
\theta_{0} \in\{\sqrt{3} \sqrt{\Lambda}\} \cup\{ \pm \sqrt{1+3 \Lambda}\} \cup\left\{\frac{1+w}{\xi_{0}}\right\}
$$

Therefore, as this calculation shows, the universe model avoids a singularity where $\theta \rightarrow \infty$ in finite time under the choice of any of these initial conditions, since with these choices the integral in Eq. (32) diverges. 
For the sake of completeness, we will also evaluate the integral in (32) for the case of a negative cosmological constant, $\Lambda<0$. In fact, let us define $\Lambda=-\lambda^{2}$, where $\lambda \neq 0 \in \mathbb{R}$. We then find that the integral in Eq. (32) has the value

$$
\begin{aligned}
& t_{s}-t_{0} \\
& =\frac{-\frac{\sqrt{3} \pi \lambda(w+1)}{|\lambda|}+3 \lambda \xi_{0}\left(2 \log \left|-\xi_{0}\right|-2 \log \left|w-\xi_{0} \theta_{0}+1\right|+\log \left|3 \lambda^{2}+\theta_{0}^{2}\right|\right)+2 \sqrt{3}(w+1) \tan ^{-1}\left(\frac{\theta_{0}}{\sqrt{3} \lambda}\right)}{3 \lambda\left(3 \lambda^{2} \xi_{0}^{2}+w^{2}+2 w+1\right)} .
\end{aligned}
$$

This has finite real values if

$$
\theta_{0}>\frac{1+w}{\xi_{0}}
$$

Therefore, for these values of $\theta_{0}$, the universe model will admit finite-time singularities.

Further, the integral in Eq. (32) will diverge if $\theta_{0}$ is chosen such that

$$
\theta_{0}=\frac{1+w}{\xi_{0}}
$$

For this choice of $\theta_{0}$, the universe model will not admit any finite-time singularities.

\section{FLRW Models With a Chaplygin Gas}

In this section, we attempt to use Osgood's criterion to determine the existence of finite-time, big crunch singularities in FLRW models with what is known as a Chaplygin gas. Unlike barotropic matter, the Chaplygin gas satisfies an exotic equation of state [4,

$$
p=-\frac{A}{\mu^{\alpha}}, \quad A \in \mathbb{R}, \quad 0<\alpha \leq 1,
$$

where $A<0$ describes an anti-Chaplygin gas, and $A>0$ describes a Chaplygin gas. Finite-time singularities as described above have been studied a number of times in the literature in the context of Chaplygin gas universes 38, 41, 63, 87.

Using Eq. (40) in Eqs. (9) and (11), we obtain the following version of Raychaudhuri's equation:

$$
\dot{\theta}=\frac{1}{2}\left[3\left(3^{\alpha} A \kappa\left(\frac{\theta^{2}-3 \Lambda}{\kappa}\right)^{-\alpha}+\Lambda\right)-\theta^{2}\right] .
$$

For simplicity, we will consider the case $\alpha=1$, thereby, obtaining

$$
\begin{aligned}
\dot{\theta} & =-\frac{\left(\theta^{2}-3 \Lambda\right)^{2}-9 A \kappa^{2}}{2\left(\theta^{2}-3 \Lambda\right)}, \\
\theta\left(t_{0}\right) & =\theta_{0} .
\end{aligned}
$$

Therefore, applying Osgood's criterion, we are to test the divergence of

$$
\int_{\theta_{0}}^{\infty} \frac{2\left(s^{2}-3 \Lambda\right)}{9 A \kappa^{2}-\left(s^{2}-3 \Lambda\right)^{2}} d s
$$


Evaluating this integral, we get

$$
t_{s}-t_{0}=-\frac{1}{2} \pi \sqrt{\frac{1}{3 \sqrt{A} \kappa-3 \Lambda}}+\frac{\tan ^{-1}\left(\frac{\theta_{0}}{\sqrt{3} \sqrt{\sqrt{A} \kappa-\Lambda}}\right)}{\sqrt{3} \sqrt{\sqrt{A} \kappa-\Lambda}}-\frac{\tanh ^{-1}\left(\frac{\theta_{0}}{\sqrt{3} \sqrt{\sqrt{A} \kappa+\Lambda}}\right)}{\sqrt{3} \sqrt{\sqrt{A} \kappa+\Lambda}} .
$$

Real and finite solutions corresponding to Eq. (45) for the case $A>0$, that is for the Chaplygin gas, occur for $\Lambda>0$ and

$$
\sqrt{A} \kappa>\Lambda, \quad 0<\theta_{0}<\sqrt{3} \sqrt{\sqrt{A} \kappa+\Lambda} .
$$

Finally, divergent solutions corresponding to Eq. (45) occur when

$$
\theta_{0}=\sqrt{3} \sqrt{\sqrt{A} \kappa+\Lambda} .
$$

As can be seen from the above calculations, finite-time singularities where $\theta \rightarrow \infty$ occur for the conditions as described in Eq. (46), while such a singularity is avoided if $\theta_{0}$ is chosen such that Eq. (47) is satisfied for $A>0$. Therefore, the finite-time singularity is avoided for a Chaplygin gas with such an initial condition.

Considering the case of an anti-Chaplygin gas, where $A<0$, we will apply Osgood's criterion by setting $A=-a^{2}$, where $a \neq 0 \in \mathbb{R}$. One can see looking at the expression in Eq. (45), no real solutions exist irrespective of the choice of principal function. Therefore, we are unable to determine singularity behaviour for an anti-Chaplygin gas. The physical interpretation of this result is perhaps that the expansion never diverges in this case.

A further interesting case occurs in the case of a Chaplygin gas, where $A>0$, but with a vanishing cosmological constant, $\Lambda=0$. In this case, applying Osgood's criterion to Eq. (44), we obtain:

$$
t_{s}-t_{0}=\frac{\tan ^{-1}\left(\frac{\theta_{0}}{\sqrt{3} \sqrt[4]{A} \sqrt{\kappa}}\right)}{\sqrt{3} \sqrt[4]{A} \sqrt{\kappa}}-\frac{\tanh ^{-1}\left(\frac{\theta_{0}}{\sqrt{3} \sqrt[4]{A} \sqrt{\kappa}}\right)}{\sqrt{3} \sqrt[4]{A} \sqrt{\kappa}}
$$

As can be seen Eq. (48) has finite and real values if

$$
0<\theta_{0}<\sqrt{3} \sqrt{\sqrt{A} \kappa} .
$$

Therefore, for these values of $\theta_{0}$, there exist finite-time singularities. Finite-time singularities for a Chaplygin gas with vanishing cosmological constant were demonstrated in 88].

Further, finite-time singularities can be avoided if $\theta_{0}$ is chosen such that

$$
\theta_{0} \in\{0\} \cup\{\sqrt{3} \sqrt{\sqrt{A} \kappa}\} .
$$

\section{On Sudden Singularities}

We now given an example of a situation where Osgood's criterion can be used to prove the existence of Type II / Sudden Singularities as described in Table1 1 above. We will consider an anti-Chaplygin gas, with $\alpha=1$ as per Eq. (40). We will further assume that the cosmological constant vanishes, $\Lambda=0$. In the case of a sudden singularity, the pressure diverges, that is, $p \rightarrow \infty$, while the energy 
density and expansion vanish. To apply Osgood's criterion to this situation, we use Eqs. (10), (11), and (40) to formulate a new initial value problem for the pressure, $p(t)$ :

$$
\begin{aligned}
\dot{p} & =-\left(\frac{a^{2} \kappa}{p}\right)^{-1 / 2} \sqrt{3} \kappa\left(a^{2}+p^{2}\right), \\
p\left(t_{0}\right) & =p_{0},
\end{aligned}
$$

where for simplicity, we have denoted the anti-Chaplygin gas constant $A$, by $A=-a^{2}$, where $a \neq 0 \in \mathbb{R}$.

Applying Osgood's criterion to the initial value problem (51)-(154), we are to evaluate

$$
t_{s}-t_{0}=\int_{\theta_{0}}^{\infty}-\frac{\left(\frac{a^{2} \kappa}{s}\right)^{1 / 2}}{\sqrt{3}\left(a^{2} \kappa+\kappa s^{2}\right)} d s
$$

We find that

$$
\begin{aligned}
t_{s} & -t_{0}= \\
& -\frac{\sqrt{a}\left[-\log \left|\sqrt{2} \sqrt{a} \sqrt{p_{0}}+a+p_{0}\right|+\log \left|-\sqrt{2} \sqrt{a} \sqrt{p_{0}}+a+p_{0}\right|-2 \tan ^{-1}\left(\frac{\sqrt{2} \sqrt{p_{0}}}{\sqrt{a}}+1\right)+2 \tan ^{-1}\left(1-\frac{\sqrt{2} \sqrt{p_{0}}}{\sqrt{a}}\right)\right]}{2 \sqrt{6} a \sqrt{\kappa}} \\
& -\frac{\pi+\log |3-2 \sqrt{2}|}{2 \sqrt{6} \sqrt{a \kappa}} .
\end{aligned}
$$

One can easily confirm that Eq. (54) is real and finite for all $p_{0} \in \mathbb{R}$. Therefore, for all $p_{0} \in \mathbb{R}$, we have that the universe model will exhibit a finite-time sudden singularity.

\section{Conclusions}

In this paper, we have applied Osgood's criterion to detect finite-time singularities in a spatially flat FLRW universe in the context of a perfect fluid, a perfect fluid with bulk viscosity, and a Chaplygin and anti-Chaplygin gas. In particular, we applied Osgood's criterion to demonstrate singularity behaviour for Type 0/big crunch singularities as well as Type II/sudden singularities. We have shown that in each case the choice of initial conditions is important as a certain number of initial conditions leads to finite-time singularities, while other precise choices of initial conditions which depend on the cosmological matter parameters and the cosmological constant can avoid a finite-time singularity.

As we have shown, Osgood's criterion provides a powerful and yet simple way of deducing the existence of these singularities, and also interestingly enough, provides clues of how to eliminate singularities from certain cosmological models. For this reason, we believe that the work here is unique, and could be of interest in future cosmological studies. 


\section{References}

[1] W. Osgood, "Beweis der existenz einer lsung der differentialgleichung $\frac{d y}{d x}=f(x, y)$ ohne hinzunahme der cauchy-lipschitz'schen bedingung," Monatshefte fr Mathematik und Physik, vol. 9, no. 1 , pp. 331-345, 1898.

[2] J. A. León, L. Peralta, and J. Villa-Morales, "An Osgood's Criterion for a Semilinear Stochastic Differential Equation," arXiv:1401.7905, pp. 1-21, 2014.

[3] V. I. Arnold, Ordinary Differential Equations. Springer, third ed., 1992.

[4] G. F. Ellis, R. Maartens, and M. A. MacCallum, Relativistic Cosmology. Cambridge University Press, first ed., 2012.

[5] R. C. Tolman and M. Ward, "On the behavior of non-static models of the universe when the cosmological term is omitted," Physical Review, vol. 39, pp. 835-843, 1932.

[6] A. Raychaudhuri, "Relativistic cosmology. I," Physical Review, vol. 98, pp. 1123-1126, 1955.

[7] R. Penrose, "Gravitational collapse and space-time singularities," Phys. Rev. Lett, vol. 14, p. $57,1965$.

[8] S. Hawking and R. Penrose, "The singularities of gravitational collapse and cosmology," Proceedings of the Royal Society of London. Series A, Mathematical and Physical Sciences, vol. 314, pp. 529-548, 1970.

[9] J. D. Bekenstein, "Exact solutions of einstein-conformal scalar equations," Annals of Physics, vol. 82 , pp. 535-547, 1974.

[10] L. Parker and S. Fulling, "Quantized matter fields and the avoidance of singularities in general relativity," Physical Review D, vol. 7, pp. 2357-2374, 1973.

[11] C. Collins and G. Ellis, "Singularities in bianchi cosmologies," Physics Reports, vol. 56, pp. $65,67-105,1979$.

[12] J. Barrow and R. Matzner, "The homogeneity and isotropy of the universe," Monthly Notices of the Royal Astronomical Society, vol. 181, pp. 719-727, 1977.

[13] J. Barrow, "On the origin of cosmic turbulence," Monthly Notices of the Royal Astronomical Society, vol. 179, pp. 47-49, 1977.

[14] L. Fernández-Jambrina, "Grand rip and grand bang/crunch cosmological singularities," Phys. Rev. D, vol. 90, p. 064014, Sept. 2014.

[15] R. R. Caldwell, M. Kamionkowski, and N. N. Weinberg, "Phantom energy and cosmic doomsday," Phys.Rev.Lett, vol. 91, p. 071301, 2003.

[16] L. Fernández-Jambrina and R. Lazkoz, "Classification of cosmological milestones," Physical Review D, vol. 74, p. 064030, Sept. 2006.

[17] J. D. Barrow, "More general sudden singularities," Classical and Quantum Gravity, vol. 21, pp. 5619-5622, Dec. 2004. 
[18] S. Nojiri and S. D. Odintsov, "Quantum escape of sudden future singularity," Physics Letters $B$, vol. 595, pp. 1-8, Aug. 2004.

[19] J. D. Barrow, "LETTER TO THE EDITOR: Sudden future singularities," Classical and Quantum Gravity, vol. 21, pp. L79-L82, June 2004.

[20] K. Lake, "LETTER TO THE EDITOR: Sudden future singularities in FLRW cosmologies," Classical and Quantum Gravity, vol. 21, pp. L129-L132, Nov. 2004.

[21] S. Nojiri and S. D. Odintsov, "Final state and thermodynamics of a dark energy universe," Phys. Rev. D, vol. 70, p. 103522, Nov. 2004.

[22] M. P. Dabrowski, "Inhomogenized sudden future singularities," Phys. Rev. D, vol. 71, p. 103505, May 2005.

[23] M. P. Dąbrowski, "Statefinders, higher-order energy conditions, and sudden future singularities [rapid communication]," Physics Letters B, vol. 625, pp. 184-188, Oct. 2005.

[24] L. P. Chimento and R. Lazkoz, "On Big Rip Singularities," Modern Physics Letters A, vol. 19, pp. 2479-2484, 2004.

[25] J. D. Barrow, A. B. Batista, J. C. Fabris, and S. Houndjo, "Quantum particle production at sudden singularities," Phys. Rev. D, vol. 78, p. 123508, Dec. 2008.

[26] J. D. Barrow and S. Z. W. Lip, "Classical stability of sudden and big rip singularities," Phys. Rev. D, vol. 80, p. 043518, Aug. 2009.

[27] S. Nojiri and S. D. Odintsov, "Future evolution and finite-time singularities in F(R) gravity unifying inflation and cosmic acceleration," Phys. Rev. D, vol. 78, p. 046006, Aug. 2008.

[28] J. D. Barrow, S. Cotsakis, and A. Tsokaros, "A general sudden cosmological singularity," Classical and Quantum Gravity, vol. 27, p. 165017, Aug. 2010.

[29] T. Denkiewicz, M. P. Dąbrowski, H. Ghodsi, and M. A. Hendry, "Cosmological tests of sudden future singularities," Phys. Rev. D, vol. 85, p. 083527, Apr. 2012.

[30] J. D. Barrow, G. J. Galloway, and F. J. Tipler, "The closed-universe recollapse conjecture," MNRAS, vol. 223, pp. 835-844, Dec. 1986.

[31] Y. Shtanov and V. Sahni, "LETTER TO THE EDITOR: New cosmological singularities in braneworld models," Classical and Quantum Gravity, vol. 19, pp. L101-L107, June 2002.

[32] V. Gorini, A. Kamenshchik, U. Moschella, and V. Pasquier, "Tachyons, scalar fields, and cosmology," Phys. Rev. D, vol. 69, p. 123512, June 2004.

[33] A. O. Barvinsky, C. Deffayet, and A. Y. Kamenshchik, "CFT driven cosmology and the DGP/CFT correspondence," JCAP, vol. 5, p. 034, May 2010.

[34] L. Fernández-Jambrina and R. Lazkoz, "Geodesic behavior of sudden future singularities," Phys. Rev. D, vol. 70, p. 121503, Dec. 2004.

[35] Z. Keresztes, L. Á. Gergely, A. Y. Kamenshchik, V. Gorini, and D. Polarski, "Will the tachyonic universe survive the big brake?," Phys. Rev. D, vol. 82, p. 123534, Dec. 2010. 
[36] Z. Keresztes, L. Á. Gergely, A. Y. Kamenshchik, V. Gorini, and D. Polarski, "Soft singularity crossing and transformation of matter properties," Phys. Rev. D, vol. 88, p. 023535, July 2013.

[37] J. D. Barrow and S. Cotsakis, "Geodesics at sudden singularities," Phys. Rev. D, vol. 88, p. 067301, Sept. 2013.

[38] M. Bouhmadi-López, P. F. González-Díaz, and P. Martín-Moruno, "Worse than a big rip?," Physics Letters B, vol. 659, pp. 1-5, Jan. 2008.

[39] J. D. Barrow and C. G. Tsagas, "New isotropic and anisotropic sudden singularities," Classical and Quantum Gravity, vol. 22, pp. 1563-1571, May 2005.

[40] V. Sahni and Y. Shtanov, "Did the universe loiter at high redshifts?," Phys. Rev. D, vol. 71, p. 084018 , Apr. 2005.

[41] M. P. Dạbrowski and T. Denkiewicz, "Barotropic index w-singularities in cosmology," Phys. Rev. D, vol. 79, p. 063521, Mar. 2009.

[42] L. Fernández-Jambrina, "w-cosmological singularities," Phys. Rev. D, vol. 82, p. 124004, Dec. 2010.

[43] F. J. Tipler, "Singularities in conformally flat spacetimes," Physics Letters A, vol. 64, pp. 8-10, Nov. 1977.

[44] A. Krolak, "Towards the proof of the cosmic censorship hypothesis," Classical and Quantum Gravity, vol. 3, pp. 267-280, May 1986.

[45] P. H. Frampton, K. J. Ludwick, and R. J. Scherrer, "The little rip," Phys. Rev. D, vol. 84, p. 063003, Sept. 2011.

[46] P. H. Frampton, K. J. Ludwick, S. Nojiri, S. D. Odintsov, and R. J. Scherrer, "Models for little rip dark energy," Physics Letters B, vol. 708, pp. 204-211, Feb. 2012.

[47] P. H. Frampton, K. J. Ludwick, and R. J. Scherrer, "Pseudo-rip: Cosmological models intermediate between the cosmological constant and the little rip," Phys. Rev. D, vol. 85, p. 083001, Apr. 2012.

[48] M. Bouhmadi-López, A. Errahmani, P. Martín-Moruno, T. Ouali, and Y. Tavakoli, "The little sibling of the big rip singularity," International Journal of Modern Physics D, vol. 24, p. 1550078, July 2015.

[49] M. P. Dabrowski, Are singularities the limits of cosmology?, pp. 101-117. Sept. 2014.

[50] E. Elizalde, S. Nojiri, and S. D. Odintsov, "Late-time cosmology in a (phantom) scalar-tensor theory: Dark energy and the cosmic speed-up," Phys. Rev. D, vol. 70, p. 043539, Aug. 2004.

[51] K. Bamba, S. Nojiri, and S. D. Odintsov, "The future of the universe in modified gravitational theories: approaching a finite-time future singularity," Journal of Cosmology and Astroparticle Physics, vol. 10, p. 45, Oct. 2008.

[52] K. Bamba, S. Capozziello, S. Nojiri, and S. D. Odintsov, "Dark energy cosmology: the equivalent description via different theoretical models and cosmography tests," Astrophysics and Space Science, vol. 342, pp. 155-228, Nov. 2012. 
[53] S. Nojiri and S. D. Odintsov, "Inhomogeneous equation of state of the universe: Phantom era, future singularity, and crossing the phantom barrier," Phys. Rev. D, vol. 72, p. 023003, July 2005.

[54] G. F. Ellis, Cargese Lectures in Physics, vol. Six. Gordon and Breach, first ed., 1973.

[55] Ø. Grøn and S. Hervik, Einstein's General Theory of Relativity: With Modern Applications in Cosmology. Springer, first ed., 2007.

[56] I. S. Kohli and M. C. Haslam, "Future asymptotic behavior of a nontilted Bianchi type IV viscous model," Phys. Rev. D, vol. 87, p. 063006, Mar 2013.

[57] A. A. Coley and R. J. van den Hoogen, "Qualitative analysis of viscous fluid cosmological models satisfying the israel-stewart theory of irreversible thermodynamics," Classical and Quantum Gravity, vol. 12, no. 8, p. 1977, 1995.

[58] J. D. Barrow, "The deflationary universe: An instability of the de Sitter universe," Physics Letters B, vol. 180, pp. 335-339, Nov. 1986.

[59] J. D. Barrow, "Deflationary universes with quadratic lagrangians," Physics Letters B, vol. 183, pp. 285-288, Jan. 1987.

[60] J. D. Barrow, "String-driven inflationary and deflationary cosmological models," Nuclear Physics B, vol. 310, pp. 743-763, Dec. 1988.

[61] J. D. Barrow, "Graduated inflationary universes.," Physics Letters B, vol. 235, pp. 40-43, Jan. 1990.

[62] V. Belinskii and I. Khalatnikov, "Influence of viscosity on the character of cosmological evolution," Soviet Physics JETP, vol. 42, p. 205, 1976.

[63] A. Borowiec, A. Stachowski, M. Szydłowski, and A. Wojnar, "Inflationary cosmology with Chaplygin gas in Palatini formalism," JCAP, vol. 1, p. 040, Jan. 2016.

[64] L. P. Chimento and M. G. Richarte, "Big brake singularity is accommodated as an exotic quintessence field," ArXiv e-prints, Dec. 2015.

[65] A. A. Mamon and S. Das, "Study of non-canonical scalar field model using various parametrizations of dark energy equation of state," European Physical Journal C, vol. 75, p. 244, June 2015.

[66] L. P. Chimento and S. Carneiro, "Interacting vacuum energy in the dark sector," in American Institute of Physics Conference Series, vol. 1647 of American Institute of Physics Conference Series, pp. 10-12, Mar. 2015.

[67] S. L. Parnovsky, "Big Rip and Other Singularities in Isotropic Homogeneous Cosmological Models with Arbitrary Equation of State," Odessa Astronomical Publications, vol. 28, p. 137, 2015.

[68] A. Y. Kamenshchik, Z. Keresztes, L. A. Gergely, V. Gorini, and D. Polarski, "Soft singularity crossing and transformation of matter properties," in American Institute of Physics Conference Series, vol. 1606 of American Institute of Physics Conference Series, pp. 79-85, July 2014. 
[69] D.-X. Kong and C. Wei, "Formation and propagation of singularities in one-dimensional Chaplygin gas," Journal of Geometry and Physics, vol. 80, pp. 58-70, June 2014.

[70] M. Bouhmadi-López, C. Kiefer, and M. Krämer, "Resolution of type IV singularities in quantum cosmology," Phys. Rev. D, vol. 89, p. 064016, Mar. 2014.

[71] S. D. Sadatian, "Rip Singularity Scenario and Bouncing Universe in a Chaplygin Gas Dark Energy Model," International Journal of Theoretical Physics, Oct. 2013.

[72] P. Rudra, C. Ranjit, and S. Kundu, "How effective is new variable modified Chaplygin gas to play the role of dark energy a dynamical system analysis in RS II brane model," Astrophysics and Space Science, vol. 347, pp. 433-444, Oct. 2013.

[73] A. Mokeeva and V. Popov, "Isotropization in Chaplygin Matter Universes Connected by a Wormhole," International Journal of Modern Physics D, vol. 22, p. 1350067, Aug. 2013.

[74] E. O. Babichev, V. I. Dokuchaev, and Y. N. Eroshenko, "Perfect fluid and scalar field in the Reissner-Nordström metric," Soviet Journal of Experimental and Theoretical Physics, vol. 112, pp. 784-793, May 2011.

[75] P. Pedram and S. Jalalzadeh, "Chaplygin gas quantum universe in the presence of the cosmological constant," General Relativity and Gravitation, vol. 42, pp. 745-762, Apr. 2010.

[76] M. Bouhmadi-López, C. Kiefer, B. Sandhöfer, and P. V. Moniz, "Quantum fate of singularities in a dark-energy dominated universe," Physical Review D, vol. 79, p. 124035, June 2009.

[77] H. Zhang, Z.-H. Zhu, and L. Yang, "Hybrid Chaplygin Gas," Modern Physics Letters A, vol. 24, pp. 541-555, 2009.

[78] V. Gorini, U. Moschella, A. Y. Kamenshchik, V. Pasquier, and A. A. Starobinsky, "TolmanOppenheimer-Volkoff equations in the presence of the Chaplygin gas: Stars and wormholelike solutions," Physical Review D, vol. 78, p. 064064, Sept. 2008.

[79] A. V. Yurov, A. V. Astashenok, and P. F. González-Díaz, "Astronomical bounds on a future Big Freeze singularity," Gravitation and Cosmology, vol. 14, pp. 205-212, Aug. 2008.

[80] G. Panotopoulos, "Comparison between different cosmological models," Physical Review D, vol. 77 , p. 107303 , May 2008.

[81] P. Pedram and S. Jalalzadeh, "Quantum FRW cosmological solutions in the presence of Chaplygin gas and perfect fluid," Physics Letters B, vol. 659, pp. 6-13, Jan. 2008.

[82] M. Bouhmadi-López, P. F. González-Díaz, and P. Martín-Moruno, "On the Generalized Chaplygin Gas:. Worse than a Big Rip or Quieter than a Sudden Singularity?," International Journal of Modern Physics D, vol. 17, pp. 2269-2290, 2008.

[83] M. Bouhmadi-López and R. Lazkoz, "Chaplygin DGP cosmologies," Physics Letters B, vol. 654, pp. 51-57, Oct. 2007.

[84] L. P. Chimento and R. Lazkoz, "Duality gives rise to Chaplygin cosmologies with a big rip," Classical and Quantum Gravity, vol. 23, pp. 3195-3203, May 2006. 
[85] V. Gorini, A. Kamenshchik, U. Moschella, V. Pasquier, and A. Starobinsky, "Stability properties of some perfect fluid cosmological models," Physical Review D, vol. 72, p. 103518, Nov. 2005.

[86] A. A. Sen and R. J. Scherrer, "Generalizing the generalized Chaplygin gas," Physical Review $D$, vol. 72 , p. 063511 , Sept. 2005.

[87] M. Bouhmadi-López and J. A. Jiménez Madrid, "Escaping the big rip?," JCAP, vol. 5, p. 005, May 2005.

[88] A. Kamenshchik, U. Moschella, and V. Pasquier, "An alternative to quintessence," Physics Letters B, vol. 511, no. 2-4, pp. 265 - 268, 2001. 